\title{
Email Spam Detection using Machine Learning Techniques
}

\author{
Rajesh Kumar $\mathbf{J}^{1}$, Sudarshan $\mathbf{P}^{2}$, Mahalakshmi $\mathbf{G}^{\mathbf{3}}$ \\ ${ }^{1}$ PG - Master of Computer Application, CEG Anna University, Chennai, Tamil Nadu \\ ${ }^{2}$ PG - Master of Computer Application, CEG Anna University, Chennai, Tamil Nadu \\ ${ }^{3}$ Teaching Fellow, Master of Computer Application, CEG Anna University, Chennai, Tamil Nadu
}

\begin{abstract}
Email spam is one of the biggest threats to today's Internet. To dealwith this threat, many anti-spam filters have been developed. One big challengefor these filters is to predict the labels of emails in a personalized mailbox. These spam messages can lead to loss of private data as well. Modern day researchershave used some stylistic features of text messages to classify them to be hamor spam. E-mail spam detection can be greatly influenced by the presence of known words, phrases, abbreviations and idioms. This paper aims to compare different classifying techniques on different datasets collected from previous research works, and evaluate them on the basis of their accuracy, recall, and precision. The comparison hasbeen performed between traditional machine learning techniques. Most of the time such emails are commercial. But many times, such emails may contain some phishing links that have malware. This arises the need for proposing prudent mechanism to detect or identify such spam emails so that time and memory space of the system can be saved up to a great extent. In this paper, we presented the NLP mechanism which can filter spam and non-spam emails and also categorize into different spam mails. Our proposed algorithm generatesdictionary and features and trains them through machine learning for effective results.
\end{abstract}

Keywords: Naive Bayes, Support Vector Machine, Natural Language Processing, analysis.

\section{INTRODUCTION}

Consider a case in which someone over the internet is sending bulk emails regarding the promotion of their products for purchasing it, or someone sending a link to click or activate to win some lucrative prizes; such emails are generally considered as negative marketing strategies or fraud activities. As a receiver, you are helpless in this scenario. These unwanted emails may consume a lot of memory of your system also waste your precise time. It is also observed that one can be distributed by receiving such bogus emails again and again. So, there is a need for some mechanism that can reduce or even provide some sort of panacea to from these spam emails. Keep this situation in mind, in this paper; we are presenting a machine learning-based spam detection mechanism that uses a dataset of approximately 6000 valid and invalid collection of emails. Our proposed model will first make a dictionary that remove helping verbs form the contents of the email. Now our proposed algorithm will run to check whether the entered email address is spam or not. By applying this mechanism, a user can work efficiently as comparatively fewer spam emails will be received. This mechanism also saves the time and memory of the system.

\section{EXPERIMENTAL METHODS OR METHODOLOGY}

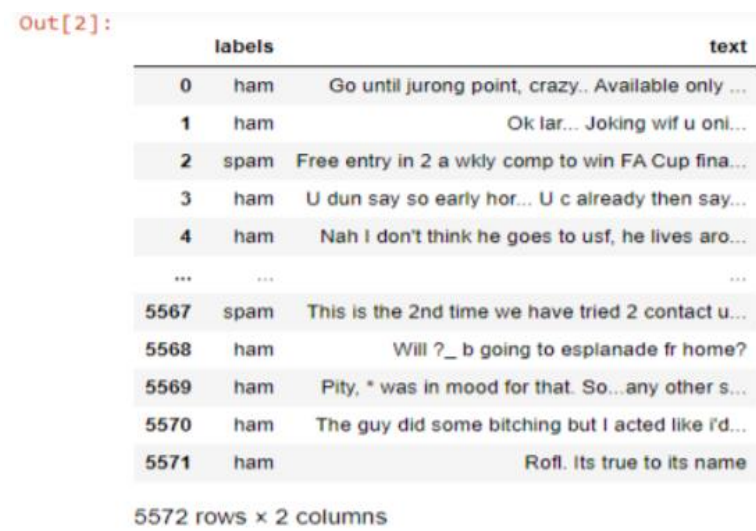

Figure 1: Email spam dataset extracted 


\section{International Advanced Research Journal in Science, Engineering and Technology}

Vol. 8, Issue 6, June 2021

DOI: $10.17148 /$ IARJSET.2021.8632

\subsection{Machine Learning Algorithms}

Machine learning is used to predict the system output easily without doing any programs. Basically, it builds the algorithm to receive the input then perform some analysis to generate the output. The expected output is going to be most accurate for the respective system.

\subsubsection{Naive Bayes Classifier}

Naive Bayes could be a reasonably classifier which uses the Bayes Theorem. It predicts membership probabilities for every class like the probability that given record or datum belongs to a specific class. The category with the very best probability is taken into account because the possibly class.

\subsubsection{Support Vector Machine}

Support Vector Machine may be a supervised machine learning algorithm which might be used for classification. Support Vectors are simply the co-ordinates of individual observation. It performs classification by finding the hyperplane that maximizes the margin between the two classes. The vectors that outline the hyperplane are the support vectors. Hyperplane separates the vectors (cases) into two non-overlapping classes. Perfect separation might not be possible.

\subsection{Natural Language Processing}

Natural language processing (NLP) is a field of artificial intelligence in which computers analyse, understand, and derive meaning from human language in a smart and useful way. By utilizing NLP, developers can organize and structure knowledge to perform tasks such as automatic summarization, translation, named entity recognition, relationship extraction, sentiment analysis, speech recognition, and topic segmentation.

\section{RESULTS AND DISCUSSION}

\subsection{Dataset Collection}

The figure 2 is referred to as the sample dataset in the csv file. Dataset Name: Spam Mails Dataset (enron-1 folder of spam dataset). This dataset is collected from Kaggle global public database repository. Used enron1 folder. Dataset Link: https://www2.aueb.gr/users/ion/data/enron-spam/

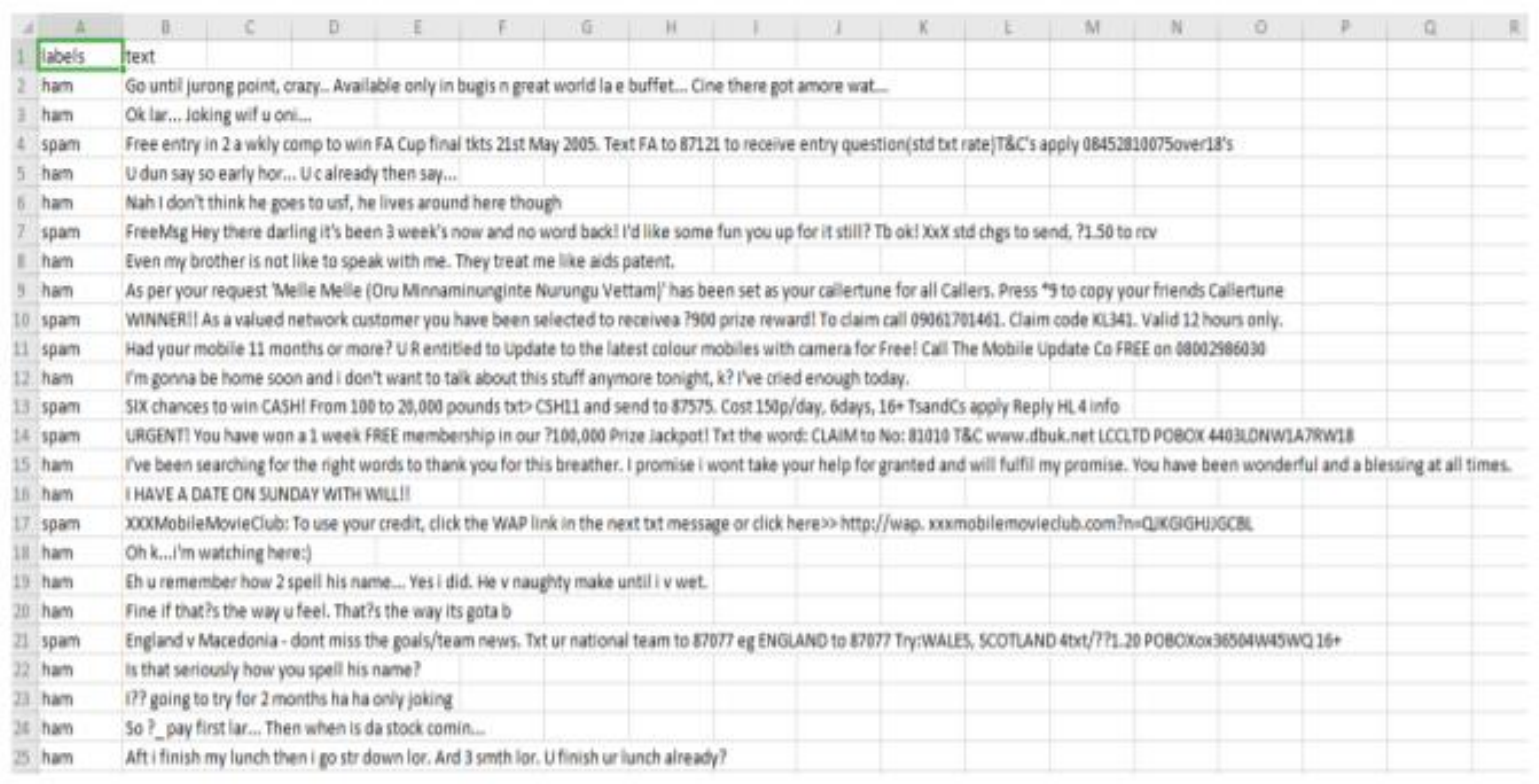

Figure 2: Datasets (in CSV) Which are Collected Using Kaggle

\subsection{Evaluation Indicators}

To evaluate the results of the two algorithms we use four of the most popular measures: Accuracy, Precision, Recall, and F1 score. These four metrics are explained in the following:

1. Accuracy Rate is the most popular measure and also very easy to understand because is a simple ratio between the number of instances correctly predicted to the total number of instances used in the observation, in other words, accuracy gives the percentage of correctly predicted instances.

2. Precision is a measure that provides for each class the ratio between correctly positive predicted instances and total of positive instances predicted.

$$
\mathbf{P}=\mathbf{T} \mathbf{P} \text { T P+FP }
$$

3. Recall is a measure that provides for each class the ratio between the true positive instances predicted and the sum of true positives and false negatives in the observation. 


\section{International Advanced Research Journal in Science, Engineering and Technology}

Vol. 8, Issue 6, June 2021

DOI: $10.17148 /$ IARJSET.2021.8632

$$
\mathbf{R}=\mathbf{T} \mathbf{P} \mathbf{T} \mathbf{P}+\mathbf{F N}
$$

4. Fl score is the weighted average of Precision and Recall and it is considered perfect when it is 1.0 and the worst possible value is 0.0 , so a good F1 score means that we have low false positives and low false negatives.

\section{$\mathbf{F}=\mathbf{2 P x R} \mathbf{P}+\mathbf{R}$}

\subsection{Classification Analysis}

The Figure 3 shows the 5572 unique email messages that is splitted into the legitimate mail and spam email using the confusion matrix heat map.

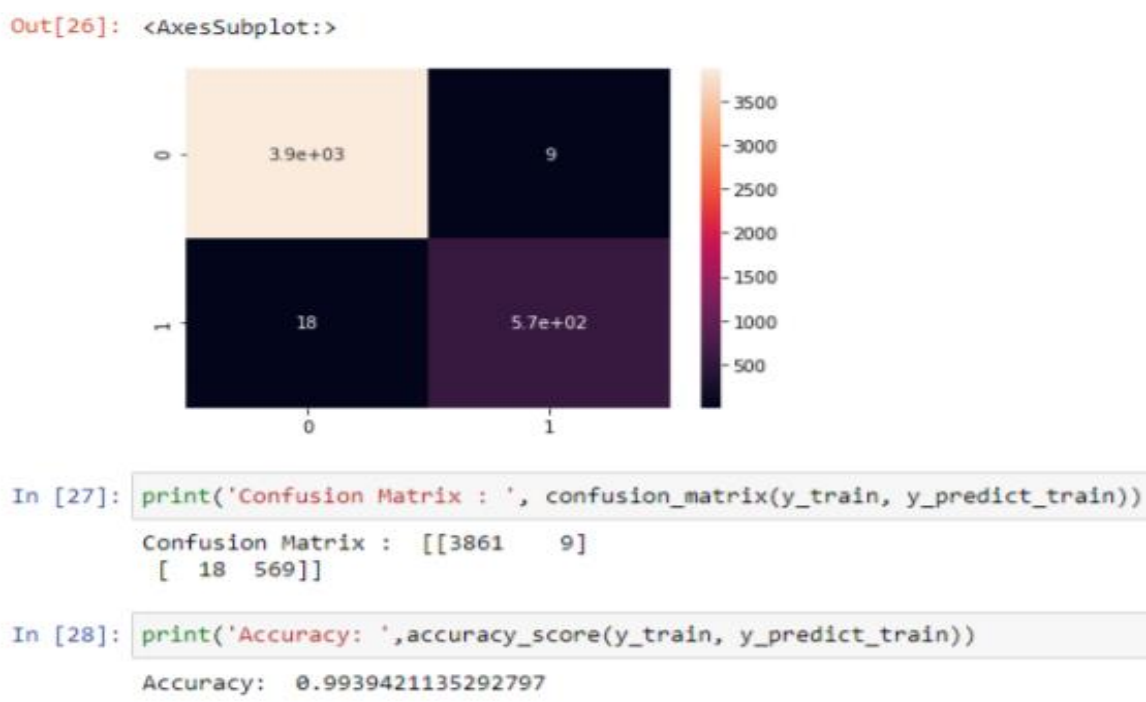

Figure 3: Legitimate and Spam mail

\subsection{Naïve Bayes and SVM Results}

\begin{tabular}{|c|c|c|c|c|c|}
\hline \multirow[t]{2}{*}{ [n $[35]:$} & \multicolumn{5}{|c|}{ print(classification_report(y_test, y_predict_test))| } \\
\hline & & precision & recall & f1-score & support \\
\hline & ham & 0.99 & 0.99 & 0.99 & 955 \\
\hline & spam & 0.92 & 0.94 & 0.93 & 160 \\
\hline & accuracy & & & 0.98 & 1115 \\
\hline & macro avg & 0.96 & 0.97 & 0.96 & 1115 \\
\hline & weighted avg & 0.98 & 0.98 & 0.98 & 1115 \\
\hline
\end{tabular}

Figure 4: Naive Bayes Results

The Figure 4 are the details on most informative features after the classifier is executed on train data using Naive Bayes.

\begin{tabular}{|c|c|c|c|c|c|}
\hline \multirow[t]{2}{*}{ In $[26]:$} & \multicolumn{5}{|c|}{ print(classification_report(y_test, y_predict_test)) } \\
\hline & & precision & recall & f1-score & suppor \\
\hline & ham & 0.97 & 1.00 & 0.98 & 95 \\
\hline & spam & 1.00 & 0.79 & 0.88 & 15 \\
\hline & accuracy & & & 0.97 & 111 \\
\hline & macro avg & 0.98 & 0.89 & 0.93 & 111 \\
\hline & weighted avg & 0.97 & 0.97 & 0.97 & 111 \\
\hline
\end{tabular}

Figure 5: SVM Results 


\section{International Advanced Research Journal in Science, Engineering and Technology}

Vol. 8, Issue 6, June 2021

DOI: $10.17148 /$ IARJSET.2021.8632

The Figure 5 are the details on most informative features after the classifier is executed on train data.

\subsection{Comparison and Evaluation Results}

The Figure 6 shows that the accuracy rate of the twitter sentiment analysis based on Bayesian network can reach 94.09 percent in the top 5000 results, and the recall rate can reach 89.35 percent, and the F- measure can reach 90.45 percent, which shows that the proposed algorithm in this paper is still relatively satisfactory results.

\begin{tabular}{|c|c|c|c|c|}
\hline Parameters & Accuracy & Precision & Recall & F-Measure \\
\hline Naive Bayes & 98 & 92 & 94 & 93 \\
\hline SVM & 97 & 100 & 79 & 88 \\
\hline
\end{tabular}

Figure 6: Evaluation Results of Two Algorithms

The above Figure 6 is a comparison with the similarity algorithm based on the SVM, the average accuracy rate is improved by 75.09 percent, the average recall rate is increased by 82.17 percent, and the average F-measure value is increased by 81.21 percent.

\subsection{Result Analysis}

Regardless of the evaluation index of the algorithm, the algorithm proposed is higher than the other algorithms. In this case, with the number of detected spam emails increasing, the accuracy decreases, and the recall rate and $F$ measurement increases. This is mainly due to the number's increase of detected text, it increased the scope of the query, so resulting in increased recall rate. With continuous expansion of the inspection range, the error will increase and accuracy rate will decrease.

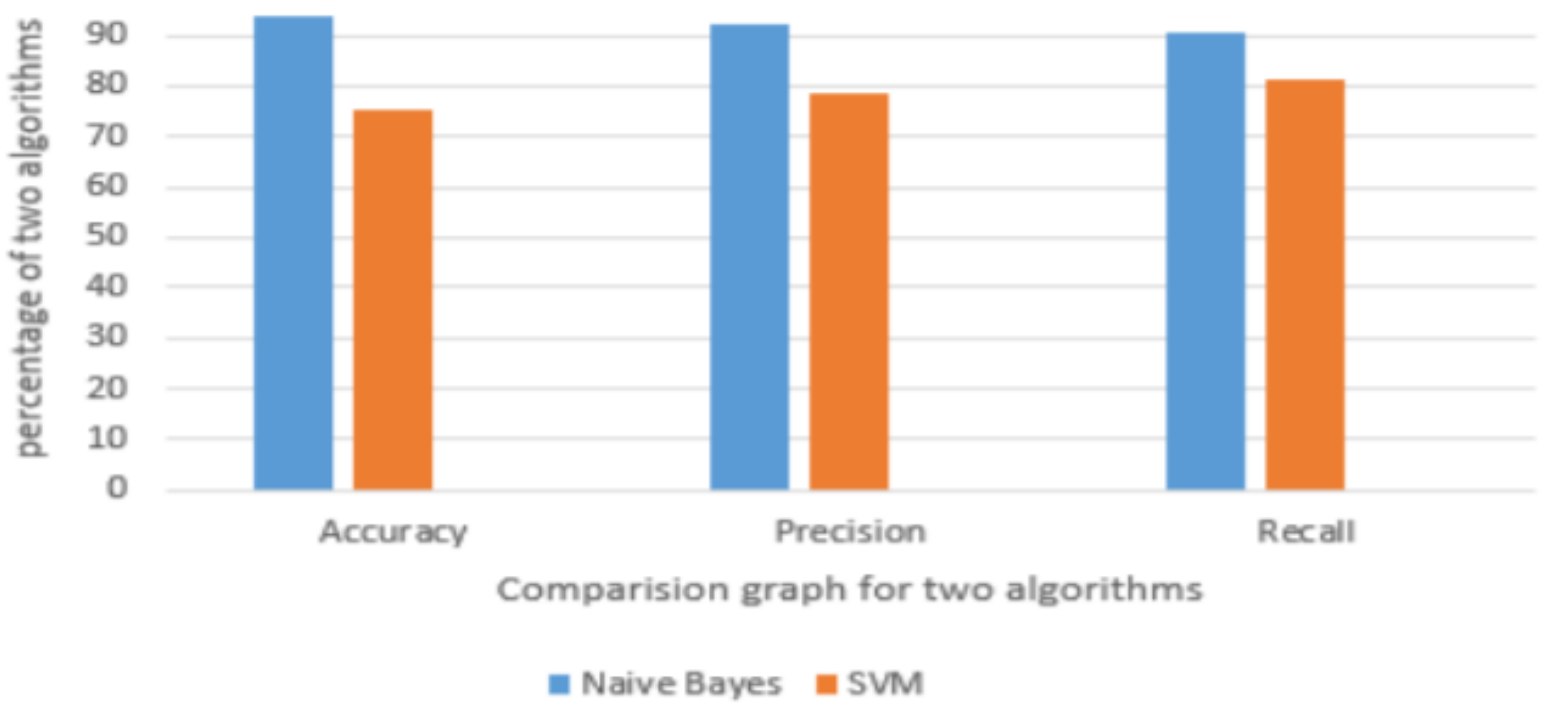

Figure 6: Graph for Comparison of Two Algorithms

It can be seen from Figure 5.9 that the accuracy rate of the similarity algorithm based on Naïve Bayes algorithm is obviously higher than that SVM network.

\section{CONCLUSION}

This Project provides a work flow to understand and detect the legitimate and spam emails . To classify email spam dataset into five predefined categories to get in-depth knowledge of their learning experiences. It shows that the results derived from the Naive Bayes classifiers are much better than that of the SVM for text classification. Mining messages for understanding the email spam texts in python. It helps in understand the machine to understand human phrases and conversations. 


\section{IARJSET}

\section{International Advanced Research Journal in Science, Engineering and Technology}

Vol. 8, Issue 6, June 2021

DOI: $10.17148 /$ IARJSET.2021.8632

\section{REFERENCES}

[1]. Sefat E Rahman and Shofi Ullah. Email spam detection using bidirectional long short-term memory with convolutional neural network. In 2020 IEEE Region 10 Symposium (TENSYMP), pages 1307-1311. IEEE, 2020.

[2]. Milivoje Popovac, Mirjana Karanovic, Srdjan Sladojevic, Marko Arsenovic, \& Andras Anderla. Convolutional neural network based sms spam detection. In $201826^{\text {th }}$ Telecommunication Forum(TELFOR), page1-4. IEEE, 2018

[3]. Nikhil Govil, Kunal Agarwal, Ashi Bansal, and Astha Varshney. A machine learning based spam detection mechanism. In 2020 Fourth International Conference on Computing Methodologies and Communication (ICCMC), pages 954-957. IEEE, 2020.

[4]. Aakash Atul Alurkar, Sourabh Bharat Ranade, Shreeya Vijay Joshi, Siddhesh Sanjay Ranade, Piyush A Sonewar, Parikshit N Mahalle, and Arvind V Deshpande. A proposed data science approach for email spam classification using machine learning techniques. In 2017 Internet of Things Business Models, Users, and Networks, pages 1-5. IEEE, 2017.

[5]. Mehul Gupta, Aditya Bakliwal, Shubhangi Agarwal, and Pulkit Mehndiratta. A comparative study of spam sms detection using machine learning classifiers. In 2018 Eleventh International Conference on Contemporary Computing (IC3), pages 1-7. IEEE, 2018.

[6]. N. Kumar, S. Sonowal, and Nishant. Email spam detection using machine learning algorithms. In 2020 Second International Conference on Inventive Research in Computing Applications (ICIRCA), pages 108-113, 2020

[7]. K. Agarwal and T. Kumar. Email spam detection using integrated approach of nä1ve bayes and particle swarm optimization. In 2018 Second International Conference on Intelligent Computing and Control Systems (ICICCS), pages 685-690, 2018.

[8]. P. Liu and T. Moh. Content based spam e-mail filtering. In 2016 International Conference on Collaboration Technologies and Systems (CTS), pages 218-224, 2016.

[9]. M. K. Chae, A. Alsadoon, P. W. C. Prasad, and A. Elchouemi. Spam filtering email classification (sfecm) using gain and graph mining algorithm. In 2017 IEEE 7th Annual Computing and Communication Workshop and Conference (CCWC), pages 1-7, 2017. 51

[10]. S. Suryawanshi, A. Goswami, and P. Patil. Email spam detection: An empirical comparative study of different ml and ensemble classifiers. In 2019 IEEE 9th International Conference on Advanced Computing (IACC), pages 69-74, 2019.

[11]. E. E. Eryilmaz, D. Ozkan S, ahin, and E. K1lic,. Machine learning based spam e-mail detection system for turkish. In 2020 5th International Conference on Computer Science and Engineering (UBMK), pages 7-12, 2020.

[12]. W. Hijawi, H. Faris, J. Alqatawna, A. M. Al-Zoubi, and I. Aljarah. Improving email spam detection using content-based feature engineering approach. In 2017 IEEE Jordan Conference on Applied Electrical Engineering and Computing Technologies (AEECT), pages 1-6, 2017.1. 